\title{
ORT_04 - Use of a theophylline responsive riboswitch for translational control applied to the expression of secreted heterologous proteins in Mycobacterium smegmatis
}

\author{
Victor Gigante Pereira ${ }^{1 *}$; Marcos Gustavo Araujo Schwarz¹; Leila Mendonça-Lima1. \\ ${ }^{1}$ Fiocruz/IOC.
}

Introduction: Recombinant DNA technology is an indispensable tool to obtain recombinant proteins in a large scale. It has several advantages when compared to native protein purification, such as reduced cost throughout production, yielding large quantities of the desired protein in a more accessible way. The most studied and used bacterial host for this purpose is Escherichia coli, but, among other disadvantages, it lacks secretory pathways to produce heterologous protein in the culture medium. So, other bacteria are being used as hosts, such as Mycobacterium smegmatis. One of the most used approaches during heterologous protein production is the control of protein expression, mainly on the transcriptional level. Nowadays, the use of riboswitches responsive to small molecules to control expression at the translational level is being investigated in several models.

Objective: Build vectors for controlled expression using riboswitches and secretion of heterologous proteins in Mycobacterium smegmatis.

Methodology: 1) Riboswitch insertion: riboswitch sequences were inserted between the promoter and signal peptide sequences from pUS976, a mycobacterial shuttle vector. Each fragment (corresponding to promoter, riboswitch and signal peptide) were separately amplified by PCR, and then fused by a second PCR round (promoter+ribo and ribo+signal peptide). In a third PCR, the whole fragment was generated. 2) Cloning: the fused fragment containing the riboswitch was cloned on the original pUS976, exchanging its own promoter and signal peptide sequence for the one with the riboswitch. To achieve that, both pUS976 and the fused PCR fragment DNA were digested with BamHI/XbaI, followed by purification and ligation with T4 DNA ligase, using manufacturer's protocol. Ligation reaction was transformed on E. coli TOP10 and resulting plasmids were sequenced, to detect the proper sequence structure.

Results: We successfully obtained pUS976 with two different riboswitch sequences between promoter and signal peptide.

Conclusion: We developed plasmids for heterologous protein secretion, with expression controlled by a theophylline responsive riboswitch. Further assays will be performed using reporter genes to test both plasmids on their ability to control protein expression on a translational level.

Keywords: recombinant protein expression; riboswitch; mycobacteria 